\title{
Pacific
}

Journal of

Mathematics

\section{ON RICCI DEFORMATION OF A RIEMANNIAN METRIC ON MANIFOLD WITH BOUNDARY}

YING SHEN

Volume 173 No. 1

March 1996 


\title{
ON RICCI DEFORMATION OF A RIEMANNIAN METRIC ON MANIFOLD WITH BOUNDARY
}

\author{
YING SHEN
}

In this paper, we applied Hamilton's Ricci flow to study the metric deformation on Riemannian manifolds with boundary. We proved a short time existence theorem for manifold with umbilical boundary. We also derived the Simons' identity for the boundary under the Ricci flow. And as a corollary, we show that any three-manifold with totally geodesic boundary which admits positive Ricci curvature can be deformed to a space form with totally geodesic boundary.

\section{Introduction.}

In 1982, R.S. Hamilton [Ham] introduced an evolution equation method which is called the Ricci flow, and he proved that every closed three-dimensional Riemannian manifold with strictly positive Ricci curvature admits a metric of constant positive curvature. His work immediately brought many mathematicians to the study of Ricci flow and other evolution equations arising from differential geometry such as mean curvature flow, Yamabe flow, Yang-Mills flow and curve-shortening. A large number of nice results in geometry and partial differential equations have been obtained since then.

In this work, we study the deformation of a Riemannian metric on a compact manifold with boundary via Hamilton's Ricci flow. From the analytic point of view, Ricci deformation of a given closed Riemannian manifold $(M, \stackrel{\circ}{g})$ :

$$
\left\{\begin{array}{l}
\frac{\partial}{\partial t} g=-2 \text { Ric } \\
g(x, 0)=\stackrel{\circ}{g}(x)
\end{array}\right.
$$

is a Cauchy problem. Therefore, it is natural to consider boundary value problems for the Ricci flow. In this paper, we only restrict ourselves to the Neuman boundary value problem. The Dirichlet boundary value problem and the mixed boundary value problem will be studied in the forthcoming papers.

We remark that the boundary conditions for the Ricci flow are quite subtle. By looking at the evolution equations

$$
\frac{\partial}{\partial t} g_{i j}=-2 R_{i j}
$$


one might try to impose $\frac{n(n-1)}{2}$ functions on the boundary and expect to obtain some geometric theorems from the resulting boundary value problem. It turns out that this is not possible. Unfortunately, we generally do not even have the short time solution of the boundary value problem mentioned above due to the fact that the equality

$$
\phi^{*}(\operatorname{Ric}(g))=\operatorname{Ric}\left(\phi^{*}(g)\right)
$$

holds for any diffeomorphism $\phi$ on $M$. Therefore, one should carefully study the geometry of the boundary in order to set up a well-posed boundary value problem.

Let $(M, g)$ be an n-dimensional manifold. We adopt the convention that Latin indices range from 1 to $\mathrm{n}$, while Greek indices range from 1 to $\mathrm{n}-1$. Now suppose that the boundary $\partial M \neq \varnothing$. Let $h=\left(h_{\alpha \beta}\right)$ be the second fundamental form of $\partial M$ in $M$. We need the following definition:

Definition. We say that $\partial M$ is weakly umbilical in $M$ if the identity

$$
h_{\alpha \beta}=\lambda g_{\alpha \beta}
$$

holds on $\partial M$, where $\lambda$ is a constant.

In case the constant $\lambda=0$, we say that $\partial M$ is totally geodesic.

Now we are going to state our main result in the paper.

Theorem 1. For any given Riemannian manifold $(M, \stackrel{\circ}{g})$, there is a short time solution to the following equations:

$$
\begin{cases}\frac{\partial}{\partial t} g_{i j}(x, t)=-2 R_{i j}(x, t) & x \in M \\ g_{i j}(x, 0)=\stackrel{\circ}{g}_{i j}(x) & x \in M \\ h_{\alpha \beta}(x, t)=\lambda g_{\alpha \beta}(x, t) & x \in \partial M .\end{cases}
$$

Theorem 2. Let $(M, g)$ be a compact three-dimentional Riemannian manifold with totally geodesic boundary and with positive Ricci curvature. Then $(M, g)$ can be deformed to $\left(M, g_{\infty}\right)$ via the Ricci flow such that $\left(M, g_{\infty}\right)$ has constant positive curvature and totally geodesic boundary.

Our proof will be presented in the following three sections. Section 2 will be devoted to showing the short time existence for the Ricci flow with Neuman boundary data. In Section 3 , we derive a parabolic version of Simons' identity [Sim]. And we will give our proof of the long time existence and convergence of the Ricci flow in Section 4.

We remark that Theorem 2 can also be proved by doubling the manifold and then applied Hamilton's result on closed three manifold. 
The author would like to thank Professor R. M. Schoen for his kind guidance and constant encouragement. He would also like to thank Professor R. S. Hamilton for his helpful discussions and encouragement.

\section{Short time existence.}

Let $(M, \stackrel{\circ}{g})$ be a given Riemannian manifold with weakly umbilical boundary such that on $\partial M$ :

$$
\stackrel{\circ}{h}=\lambda \stackrel{\circ}{g},
$$

where $\stackrel{\circ}{g}$ is the fundamental form and $\lambda$ be a constant. We are going to solve the following system:

$$
\begin{cases}\frac{\partial}{\partial t} g_{i j}(x, t)=-2 R_{i j}(x, t) & x \in M \\ g_{i j}(x, 0)=\stackrel{\circ}{g}_{i j}(x) & x \in M \\ h_{\alpha \beta}(x, t)=\lambda g_{\alpha \beta}(x, t) & x \in \partial M\end{cases}
$$

As is well-known, this system is not strictly parabolic. Therefore, we use DeTurck's trick (see also [Sc] and [Shi]) to relate system (2.4) to a modified system which is strictly parabolic with boundary and initial data that satisfy the necessary "complimentary conditions" (see [Lady]).

Before we introduce the new system, we need the following lemma:

Lemma 2.1. Let $\hat{g}$ be any background metric on $M$ with Ricci curvature $\hat{R} i c$, then we have

$$
-2 \operatorname{Ric}+2 \hat{R} i c=T(g)-L_{X} g .
$$

Where $X$ is the tension field of the identity map from $(M, \hat{g})$ to $(M, g)$, the covariant derivative 'I' is taken with respect to $\hat{g}$ and

$$
T_{i j}(g)=g^{k l} g_{i j \mid k l}+q_{i j},
$$

where $q_{i j}$ involves only the lower order terms.

Proof. We use $\Gamma_{i j}^{k}$ and $\hat{\Gamma}_{i j}^{k}$ to denote the Christoffel symbols of $g$ and $\hat{g}$, respectively. Then we have

$$
R_{i j}=\Gamma_{i j, k}^{k}-\Gamma_{k i, j}^{k}+\Gamma_{k l}^{k} \Gamma_{i j}^{l}-\Gamma_{k i}^{l} \Gamma_{j l}^{k}
$$

and

$$
\hat{R}_{i j}=\hat{\Gamma}_{i j, k}^{k}-\hat{\Gamma}_{k i, j}^{k}+\hat{\Gamma}_{k l}^{k} \hat{\Gamma}_{i j}^{l}-\hat{\Gamma}_{k i}^{l} \hat{\Gamma}_{j l}^{k}
$$

where $^{6}$,' denotes the partial derivative and repeated indices are summed. 
Since

$$
B_{i j}^{k}=\Gamma_{i j}^{k}-\hat{\Gamma}_{i j}^{k}
$$

is a well-defined tensor, we have

$$
\begin{aligned}
R_{i j}-\hat{R}_{i j}= & B_{i j \mid k}^{k}-B_{k i \mid j}^{k}+B_{k l}^{k} B_{i j}^{l}-B_{j l}^{k} B_{k i}^{l} \\
& =-\frac{1}{2} g^{k l} g_{i j \mid k l}-\frac{1}{2} g^{k l} g_{k l \mid i j}+\frac{1}{2} g^{k l}\left(g_{l i \mid j k}+g_{l j \mid i k}\right)+Q_{i j}
\end{aligned}
$$

where $Q$ is the two tensor that consists only of the lower order terms of $g$ with coefficients involving $\hat{g}$ and its derivatives.

Define the vector field such that its components are given by

$$
X^{k}=\sqrt{\frac{|g|}{|\hat{g}|}}\left(\sqrt{\frac{|\hat{g}|}{|g|}} g^{i k}\right)_{\mid i} .
$$

Now we can easily finish the rest of the proof.

We would like to mention that the vector field $X$ can also be written as

$$
X^{k}=g_{\mid i}^{i k}-\frac{1}{2} g^{i k} g^{a b} g_{a b \mid i}=g^{i j}\left(\Gamma_{i j}^{k}-\hat{\Gamma}_{i j}^{k}\right) .
$$

So it is the tension field of the identity map.

Now we are going to solve the following modified system:

$$
\begin{cases}\frac{\partial}{\partial t} g_{i j}(x, t)=T_{i j}(x, t)-2 \hat{R}_{i j}(x, t) & x \in M \\ g_{i j}(x, 0)=\stackrel{\circ}{i j}_{i j}(x) & x \in M \\ h_{\alpha \beta}(x, t)=\lambda g_{\alpha \beta}(x, t) & x \in \partial M \\ g_{n \alpha}(x, t)=0 & x \in \partial M \\ X^{n}(x, t)=0 & x \in \partial M .\end{cases}
$$

Since $\partial M$ is a hypersurface in $M$, we can choose a local coordinate chart around a given point $x \in \partial M$ such that $\left\{\frac{\partial}{\partial x^{\alpha}}\right\}$ form basis for $T_{x} \partial M$ and $\frac{\partial}{\partial x^{n}}$ is transversal to $T_{x} \partial M$. Therefore, it is easy to check that

$$
e_{n}=\frac{g^{n i}}{\left(g^{n n}\right)^{\frac{1}{2}}} \frac{\partial}{\partial x^{i}}
$$

is the unit vector that is normal to $T_{x} \partial M$ in a small neighborhood of $x$. Hence the second fundamental form of $\left(\partial M,\left.g\right|_{\partial M}\right)$ in $(M, g)$ is

$$
h_{\alpha \beta}=\frac{1}{2} \frac{g^{n i}}{\left(g^{n n}\right)^{\frac{1}{2}}}\left(g_{i \alpha \mid \beta}+g_{i \beta \mid \alpha}-g_{\alpha \beta \mid i}\right) .
$$


The given boundary condition $g_{n \alpha}=0$ implies that $g^{n \alpha}=0$ and

$$
h_{\alpha \beta}=-\frac{1}{2}\left(g^{n n}\right)^{\frac{1}{2}} g_{\alpha \beta \mid n} .
$$

By definition, we know that

$$
\begin{aligned}
X^{n} & =g^{i j} g^{n k}\left(g_{k i \mid j}-\frac{1}{2} g_{i j \mid k}\right) \\
& =g^{i j} g^{n n}\left(g_{n i \mid j}-\frac{1}{2} g_{i j \mid n}\right) \\
& =-\frac{1}{2} g^{\alpha \beta} g^{n n} g_{\alpha \beta \mid n}+\frac{1}{2}\left(g^{n n}\right)^{2} g_{n n \mid n} .
\end{aligned}
$$

Hence the condition $X^{n}=0$ is equivalent to

$$
g_{n n \mid n}=\frac{-2 g^{\alpha \beta} g_{\alpha \beta}}{\left(g^{n n}\right)^{\frac{3}{2}}} \lambda .
$$

Therefore, system (2.11) is equivalent to

$$
\begin{cases}\frac{\partial}{\partial t} g_{i j}(x, t)=T_{i j}(x, t)-2 \hat{R}_{i j}(x, t) & x \in M \\ g_{i j}(x, 0)=g_{i j}(x) & x \in M \\ g_{\alpha \beta \mid n}(x, t)=-\frac{2 \lambda}{\left(g^{n n}\right)^{\frac{1}{2}}} g_{\alpha \beta}(x, t) & x \in \partial M \\ g_{n \alpha}(x, t)=0 & x \in \partial M \\ g_{n n \mid n}=\frac{-2 g^{\alpha \beta} g_{\alpha \beta}}{\left(g^{n n}\right)^{\frac{3}{2}}} \lambda & x \in \partial M .\end{cases}
$$

Now we see immediately that (2.15) has a short time solution, and so does (2.11).

Let $g(x, t)$ be a solution of $(2.11)$, then the ordinary differential equations

$$
\left\{\begin{array}{l}
\frac{\partial}{\partial t} \phi_{t}(x)=X(\phi(x), t) \\
\phi_{0}=i d
\end{array}\right.
$$

determines a one-parameter family of diffeomorphism $y=\phi_{t}(x)$ and

$$
\phi_{t}: M \rightarrow M
$$

for small $t$, where $i d$ denotes the identity map on $M$ and the vector field $X$ has components $X^{k}$ for $k=1,2, \cdots, n$ as defined by Lemma 2.1.

Let $\bar{g}(y, t)=\left(\phi_{t}^{-1}\right)^{*}(g(x, t))$, then we can easily check that

$$
\begin{aligned}
\frac{\partial}{\partial t} \bar{g}(y, t) & =\left(\phi_{t}^{-1}\right)^{*}\left(\frac{\partial}{\partial t} g(x, t)\right)-L_{X}(\bar{g}) \\
& =\left(\phi_{t}^{-1}\right)^{*}\left(-2 \operatorname{Ric}(g)+L_{X} g\right)-L_{X}(\bar{g}) \\
& =-2 \operatorname{Ric}(\bar{g})(y, t) .
\end{aligned}
$$


Let $\bar{h}$ be the second fundamental form of $\left(\partial M,\left.\bar{g}\right|_{\partial M}\right)$ in $(M, \bar{g})$, then we have

$$
\bar{h}_{\alpha \beta}=\left(\left(\phi^{-1}\right)^{*}(h)\right)_{\alpha \beta}=\lambda \bar{g}_{\alpha \beta}
$$

from the fact that $X^{n}=0$ on $\partial M$. Therefore, we have proved that (2.4) has a short time solution.

\section{A parabolic version of Simons' identity.}

In this section, we set up the Simons-type identity which is of its own interest to study other boundary value problems for the Ricci flow. Since the metric is changing as time evolves, the orthonormal basis that we use in the study of submanifolds will not be time independent and hence the moving frame method should be applied carefully. We are going to derive our evolution equation for the fundamental form of the boundary by using a non-orthogonal moving frame.

We remark here that the formula that we establish in this section also applies to any hypersurface in $M$.

The evolution we consider is $\frac{\partial g}{\partial t}=-2 \operatorname{Ric}(g)+2 c(t) g$, where $c(t)$ is any function of $t$. Let $\left\{e_{1}, e_{2}, \cdots, e_{n}\right\}$ be any moving frame such that, restriced to $\partial M$, the vectors $e_{1}, e_{2}, \cdots, e_{n-1}$ are tangent to $\partial M$. By choosing the unit normal

$$
\hat{e}_{n}=\frac{g^{n i} e_{i}}{\left(g^{n n}\right)^{\frac{1}{2}}}
$$

we can easily check that

$$
h_{\alpha \beta}=\left(g^{n n}\right)^{-\frac{1}{2}} \Gamma_{\alpha \beta}^{n}
$$

Therefore,

$$
\begin{aligned}
\frac{\partial}{\partial t} h_{\alpha \beta} & =\left(\frac{\partial}{\partial t}\left(g^{n n}\right)^{-\frac{1}{2}}\right) \Gamma_{\alpha \beta}^{n}+\left(g^{n n}\right)^{-\frac{1}{2}} \dot{\Gamma}_{\alpha \beta}^{n} \\
& =-\frac{R^{n n}}{g^{n n}} h_{\alpha \beta}+\frac{1}{2}\left(g^{n n}\right)^{-\frac{1}{2}} g_{n i}\left(\dot{g}_{\alpha i ; \beta}+\dot{g}_{\beta i ; \alpha}-\dot{g}_{\alpha \beta ; i}\right)+c(t) h_{\alpha \beta} \\
& =-\frac{R^{n n}}{g^{n n}} h_{\alpha \beta}-\frac{g^{n i}}{\left(g^{n n}\right)^{\frac{1}{2}}}\left(R_{i \alpha ; \beta}+R_{i \beta ; \alpha}-R_{\alpha \beta ; i}\right)+c(t) h_{\alpha \beta} .
\end{aligned}
$$

If we denote $R_{\hat{n} \alpha}=R\left(\hat{e}_{n}, e_{\alpha}\right)$, then we have by straight forward computation:

$$
\frac{g^{n i}}{\left(g^{n n}\right)^{\frac{1}{2}}}\left(R_{i \alpha ; \beta}+R_{i \beta ; \alpha}+R_{\alpha \beta ; i}\right)=R_{\hat{n} \alpha ; \beta}+R_{\hat{n} \beta ; \alpha}-R_{\alpha \beta ; \hat{n}}
$$


So

$$
\frac{\partial}{\partial t} h_{\alpha \beta}=-\frac{R^{n n}}{g^{n n}} h_{\alpha \beta}-\left(R_{\hat{n} \alpha ; \beta}+R_{\hat{n} \beta ; \alpha}-R_{\alpha \beta ; \hat{n}}\right)+c(t) h_{\alpha \beta} .
$$

Now for a fixed time $t$, we may assume, without loss of generality, $e_{n}=\hat{e}_{n}$ and take $\left\{\omega^{1}, \cdots, \omega^{n}\right\}$ as the dual frame of $\left\{e_{1}, \cdots, e_{n}\right\}$.Then $\omega^{n}=0$ on $\partial M_{t}$.

If we denote $R_{i j k l}$ and $K_{\alpha \beta \gamma \delta}$ as curvature tensors of $M$ and $\partial M$ respectively, then we have the following structure equations

$$
\left\{\begin{array}{l}
d \omega^{i}=-\omega_{j}^{i} \wedge \omega^{j} \\
\frac{1}{2} R_{j k l}^{i} \omega^{k} \omega^{l}=\Omega_{j}^{i}=d \omega_{j}^{i}+\omega_{k}^{i} \wedge \omega_{j}^{k} \\
d g_{i j}=g_{i k} \omega_{j}^{k}+g_{j l} \omega_{i}^{l}
\end{array}\right.
$$

and

$$
\left\{\begin{array}{l}
d \omega^{\alpha}=-\omega_{\beta}^{\alpha} \wedge \omega^{\beta} \\
\frac{1}{2} K_{\beta \gamma \delta}^{\alpha} \omega^{\gamma} \wedge \omega^{\delta}=\Theta_{\beta}^{\alpha}=d \omega_{\beta}^{\alpha}+\omega_{\gamma}^{\alpha} \wedge \omega_{\beta}^{\gamma} \\
d g_{\alpha \beta}=g_{\alpha \gamma} \omega_{\beta}^{\gamma}+g_{\gamma \beta} \omega_{\alpha}^{\gamma}
\end{array}\right.
$$

where $\Omega_{j}^{i}$ and $\Theta_{\beta}^{\alpha}$ are curvature forms of $M$ and $\partial M$ respectively. We know, when restricting to $\partial M$,

$$
0=d \omega^{n}=-\omega_{\alpha}^{n} \wedge \omega^{\alpha}
$$

so Cartan's lemma implies: $\omega_{\alpha}^{n}=h_{\alpha \beta} \omega^{\beta}, h_{\beta \alpha}=h_{\alpha \beta}$ and

$$
\amalg_{\alpha \beta}=h_{\alpha \beta} \omega^{\alpha} \omega^{\beta}
$$

is just the second fundamental form of $\partial M$.

Since $D e_{i}=\omega_{i}^{j} e_{j}$ and $g\left(e_{n}, e_{\alpha}\right)=0$, we have

$$
\begin{aligned}
0=D\left(g\left(e_{n}, e_{\alpha}\right)\right) & =g\left(\omega_{n}^{j} e_{j}, e_{\alpha}\right)+g\left(e_{n}, \omega_{\alpha}^{i} e_{i}\right) \\
& =\omega_{n}^{\beta} g_{\alpha \beta}+\omega_{\alpha}^{n},
\end{aligned}
$$

hence

$$
\omega_{\alpha}^{n}=-\omega_{n}^{\beta} g_{\alpha \beta}
$$

or

$$
\omega_{n}^{\alpha}=-g_{\alpha}^{\beta} \omega_{\beta}^{n}=-g_{\alpha}^{\beta} h_{\beta \gamma} \omega^{\gamma} .
$$

From the structure equations, we also have

$$
\frac{1}{2} R_{\beta \gamma \delta}^{\alpha} \omega^{\gamma} \omega^{\delta}=\frac{1}{2} K_{\beta \gamma \delta}^{\alpha} \omega^{\gamma} \omega^{\delta}+\omega_{n}^{\alpha} \wedge \omega_{\beta}^{n} .
$$


Therefore

$$
K_{\beta \gamma \delta}^{\alpha}=R_{\beta \gamma \delta}^{\alpha}-g^{\alpha \eta} h_{\eta \delta} h_{\beta \delta}+g^{\alpha \eta} h_{\eta \gamma} h_{\beta \delta} .
$$

Now exterior differentiate

$$
\omega_{\alpha}^{n}=h_{\alpha \beta} \omega^{\gamma}
$$

We have

$$
d \omega_{\alpha}^{\alpha}=-\omega_{i}^{n} \wedge \omega_{\alpha}^{i}+\frac{1}{2} R_{\alpha \beta \gamma}^{n} \omega^{\beta} \wedge \omega^{\gamma}
$$

and

$$
d\left(h_{\alpha \beta} \omega^{\beta}\right)=d h_{\alpha \beta} \wedge \omega^{\beta}-h_{\alpha \beta} \omega_{\gamma}^{\beta} \wedge \omega^{\gamma} .
$$

Define

$$
h_{\alpha \beta \gamma} \omega^{\gamma}=D h_{\alpha \beta}=d h_{\alpha \beta}-h_{\alpha \gamma} \omega_{\beta}^{\gamma}-h_{\beta \gamma} \omega_{\alpha}^{\gamma},
$$

then we have

$$
\left(\frac{1}{2} R_{\alpha \beta \gamma}^{n}+h_{\alpha \beta \gamma}\right) \omega^{\beta} \omega^{\gamma}=0
$$

which implies

$$
R_{\alpha \beta \gamma}^{n}=h_{\alpha \gamma \beta}-h_{\alpha \beta \gamma}
$$

or

$$
R_{n \alpha \beta \gamma}=h_{\alpha \gamma \beta}-h_{\alpha \beta \gamma} .
$$

Next, we differentiate

$$
h_{\alpha \beta \gamma} \omega^{\gamma}=d h_{\alpha \beta}-h_{\alpha \gamma} \omega_{\beta}^{\gamma}-h_{\beta \gamma} \omega_{\alpha}^{\gamma}
$$

and define

$$
h_{\alpha \beta \gamma \delta} \omega^{\delta}=D h_{\alpha \beta \gamma}=d h_{\alpha \beta \gamma}-h_{\delta \beta \gamma} \omega_{\alpha}^{\delta}-h_{\alpha \delta \gamma} \omega_{\beta}^{\delta}-h_{\alpha \beta \delta} \omega_{\gamma}^{\delta} .
$$

Then

$$
h_{\alpha \beta \gamma \delta}-h_{\alpha \beta \delta \gamma}=K_{\beta \gamma \delta}^{\eta} h_{\eta \alpha}+K_{\alpha \beta \gamma}^{\eta} h_{\eta \beta} .
$$


Also, by restricting the covariant derivative of $R_{\alpha \beta \gamma \delta}$ to $M$, we have

$$
R_{n \alpha \beta \gamma ; \delta}=R_{n \alpha \beta \gamma \delta}-R_{n \alpha n \gamma} h_{\beta \delta}-R_{n \alpha \beta n} h_{\gamma \delta}+R_{\alpha \beta \gamma}^{\eta} h_{\eta \delta}
$$

where $R_{n \alpha \beta \gamma \delta}$ is defined by

$$
R_{n \alpha \beta \gamma \delta} \omega^{\delta}=d R_{n \alpha \beta \gamma}-R_{n \delta \beta \gamma} \omega_{\alpha}^{\delta}-R_{n \alpha \delta \gamma} \omega_{\beta}^{\delta}-R_{n \alpha \beta \delta} \omega_{\gamma}^{\delta} .
$$

Now we have

$$
\begin{aligned}
\Delta h_{\alpha \beta}=g^{\gamma \delta} h_{\alpha \beta \gamma \delta} & =g^{\gamma \delta}\left(h_{\alpha \gamma \beta \delta}-R_{n \alpha \beta \gamma \delta}\right) \\
& =g^{\gamma \delta}\left(h_{\gamma \alpha \beta \delta}-R_{n \alpha \beta \gamma \delta}\right) .
\end{aligned}
$$

By applying equation (3.38) and the Codazzi equation (3.36), we have

$$
h_{\gamma \alpha \beta \delta}=h_{\gamma \delta \alpha \beta}-R_{n \gamma \alpha \delta \beta}+K_{\alpha \beta \gamma}^{\eta} h_{\eta \gamma}+R_{\gamma \beta \delta}^{\eta} h_{\eta \alpha} .
$$

So

$$
\Delta h_{\alpha \beta}=H_{\alpha \beta}-g^{\gamma \delta}\left(R_{n \gamma \alpha \delta ; \beta}+R_{n \alpha \beta \gamma ; \delta}\right)+g^{\gamma \delta}\left(K_{\alpha \beta \delta}^{\eta} h_{\eta \gamma}+K_{\gamma \beta \delta}^{\eta} h_{\eta \alpha}\right),
$$

where the scalar $H=g^{\alpha \beta} h_{\alpha \beta}$ is the mean curvature of $\partial M$.

Using equations (3.30) and (3.42), we then obtain:

$$
\begin{aligned}
\Delta h_{\alpha \beta}=H_{\alpha \beta} & -g^{\gamma \delta}\left(R_{n \gamma \alpha \delta ; \beta}+R_{n \alpha \beta \gamma ; \delta}\right)-\left(H R_{n \alpha \beta n}+g^{\gamma \delta} h_{\alpha \beta} R_{n \gamma n \delta}\right) \\
& +g^{\gamma \delta}\left(R_{\gamma \alpha \delta}^{\eta} h_{\eta \beta}+R_{\gamma \beta \delta}^{\eta} h_{\eta \alpha}+2 R_{\alpha \beta \gamma}^{\eta} h_{\eta \delta}\right) \\
& +\left(h_{\alpha}^{\eta} h_{\eta \beta} H+h_{\gamma}^{\eta} h_{\eta \beta} h_{\alpha}^{\gamma}-h_{\gamma \eta} h^{\gamma \eta} h_{\alpha \beta}-h_{\eta \alpha} h_{\gamma}^{\eta} h_{\beta}^{\gamma}\right) .
\end{aligned}
$$

We can also derive, by a straightforward computation, that

$$
R_{\alpha \beta ; n}=R_{n \beta ; \alpha}-g^{\gamma \delta} R_{n \alpha \beta \gamma ; \delta}+R_{\alpha n \beta n ; n} .
$$

So we have

$$
\begin{aligned}
\frac{\partial}{\partial t} h_{\alpha \beta}= & -R_{n n} h_{\alpha \beta}-R_{\alpha n ; \beta}+R_{\alpha \beta ; n}-R_{n \beta ; \alpha}+c h_{\alpha \beta} \\
= & \left(-R_{n n} h_{\alpha \beta}+R_{\alpha n \beta n ; n}-R_{n \alpha ; \beta}\right)-g^{\gamma \delta} R_{n \alpha \beta \gamma ; \delta}+c h_{\alpha \beta} \\
= & \Delta h_{\alpha \beta}-H_{\alpha \beta}+H R_{n \alpha \beta n}-g^{\gamma \delta}\left(R_{\gamma \alpha \delta}^{\eta} h_{\eta \beta}+R_{\gamma \beta \delta}^{\eta} h_{\eta \alpha}\right. \\
& \left.+2 R_{\alpha \beta \gamma}^{\eta} h_{\eta \delta}\right)-h_{\alpha}^{\eta} h_{\eta \beta} H+h_{\gamma \eta} h^{\gamma \eta} h_{\alpha \beta}+R_{\alpha n \beta n ; n}+c h_{\alpha \beta} .
\end{aligned}
$$


We notice that equation (3.46) is derived under the assumption that $g_{n \alpha}=$ $\delta_{n \alpha}$ at point $(x, t) \in \partial M_{t}$. Generally, we have:

$$
\begin{aligned}
\frac{\partial}{\partial t} h_{\alpha \beta}= & \Delta h_{\alpha \beta}-H_{\alpha \beta}+H R_{\hat{n} \alpha \beta \hat{n}}-g^{\gamma \delta}\left(R_{\gamma \alpha \delta}^{\eta} h_{\eta \beta}+R_{\gamma \beta \delta}^{\eta} h_{\eta \alpha}\right. \\
& \left.+2 R_{\alpha \beta \gamma}^{\eta} h_{\eta \delta}\right)+\left(|A|^{2} h_{\alpha \beta}-h_{\alpha}^{\eta} h_{\eta \beta} H\right)+R_{\alpha \hat{n} \beta \hat{n} ; \hat{n}}+c h_{\alpha \beta}
\end{aligned}
$$

where $|A|$ is the norm of the second fundamental form.

Beginning with the Simons-type identity established above, we see that we can compute $R_{\hat{n} \alpha \hat{n} \beta ; \hat{n}}$ in terms of the other quantities on the boundary. And this implies that:

$$
R_{\hat{n} \hat{n} ; \hat{n}}=g^{\alpha \beta} \frac{\partial}{\partial t} h_{\alpha \beta}+H R_{\hat{n} \hat{n}} .
$$

Also, by restricting the covariant derivative of $R_{\alpha \beta}$ to $\partial M$, we have

$$
R_{\hat{n} \beta ; \alpha}=R_{\hat{n} \beta \alpha}-R_{\hat{n} \hat{n}} h_{\alpha \beta}+R_{\beta}^{\eta} h_{\eta \alpha} .
$$

So by the Bianchi identity, we can see that

$$
R_{\hat{n}}=2 g^{\alpha \beta} \frac{\partial}{\partial t} h_{\alpha \beta}+2 g^{\alpha \beta} R_{\hat{n} \alpha \beta}+2 R_{\beta}^{\eta} h_{\eta}^{\beta} .
$$

Also, $R_{\alpha \beta ; \hat{n}}$ can be computed from equation (3.45).

\section{Long time convergence of the metric.}

We assume in this section that the boundary is totally geodesic. Let us first list the evolution equations for all three curvature quantities which will be the basis for the a priori estimates we need for proving the convergence of the metric. We refer the readers to [Ham] for details.

$$
\begin{aligned}
\frac{\partial}{\partial t} R_{i j k l}=\Delta R_{i j k l} & +2\left(B_{i j k l}-B_{i j l k}+B_{i k j l}-B_{i l j k}\right) \\
- & g^{p q}\left(R_{p j k l} R_{q i}+R_{i p k l} R_{q j}+R_{i j p l} R_{q k}+R_{i j k p} R_{q l}\right)
\end{aligned}
$$

where $B_{i j k l}=g^{p r} g^{q s} R_{p i q j} R_{r k s l}$.

$$
\frac{\partial}{\partial t} R_{i j}=\Delta R_{i j}+2 R_{i k j l} R^{k l}-2 R_{p i} R_{j}^{p}
$$

$$
\frac{\partial}{\partial t} R=\Delta R+2|\operatorname{Ric}|^{2}
$$


In order to show the long time existence for the Ricci flow, we certainly need to calculate the related quantities at the boundary. Since we assume that $h=0$ on $M$, we have from the Simons-type identity that we derived in the last section,

$$
R_{\hat{n} \alpha \hat{n} \beta ; \hat{n}}=0 .
$$

From Codazzi equation, we know that

$$
R_{\hat{n} \alpha \beta \gamma}=0 .
$$

Also, from equation (4.51), we have

$$
R_{\hat{n} \alpha \beta \gamma ; \delta}=0 .
$$

Therefore, we have

$$
R_{\alpha \beta \gamma \delta ; \hat{n}}=0
$$

and

$$
R_{\hat{n} \beta ; \delta}=0,
$$

and by a direct calculation and by using the equation (3.45),

$$
R_{\alpha \beta ; \hat{n}}=0 .
$$

Also, we can easily see that

$$
R_{\hat{n} \hat{n} ; \hat{n}}=0
$$

and

$$
R_{\alpha \beta \gamma \delta ; \hat{n}}=0
$$

$$
R_{\hat{n} \hat{n} ; \hat{n}}=0 .
$$

Therefore, we conclude that the normal derivative of all curvature quantities vanishes on the boundary.

The next lemma, which is similar to the Hopf maximum principle for functions will be used to prove the long time existence theorem.

In order to state the lemma, we need the following definition:

Definition. Let $N_{\imath j}=p\left(M_{i j}, g_{\imath j}\right)$ be a polynomial in $M_{i j}$ obtained by contracting products of $M_{i j}$ with itself using the metric. We say that the 
$N_{i j}$ satisfy the null-eigenvector condition if $N_{i j} v^{i} v^{j} \geq 0$ for any $v^{i}$ which is a null-eigenvector of $M_{i j}$.

Lemma 4.1. Let $(M, g)$ be a Riemannian manifold such that $R_{i j} \geq-(n-$ 1) $\lambda g_{i j}$. Suppose we have

$$
\frac{\partial}{\partial t} M_{i j}=\Delta M_{i j}+u^{k} \nabla_{k} M_{i j}+N_{i j}
$$

for some constant $\lambda \geq 0$, where $N=P\left(M_{i j}, g_{i j}\right)$ satisfies the null-eigenvector condition. If $M_{i j ; \hat{n}} \geq 0$ with respect to outward normal, and $u^{k} \nabla_{k} M_{i j}=0$ at any point of minimum eigenvalue of $M_{i j}$, then the condition $M_{i j} \geq 0$ is preserved under the flow.

Proof. We need to show that $M_{i j} \geq 0$ on $0 \leq t \leq \delta$ where $\delta$ is small compared to a constant $C$ which only depends on $\left|M_{i j}\right|$, as we shall see below. The repeated application of this procedure will yield the result.

Let $\hat{M}_{i j}=M_{i j}+\epsilon(\delta+t) g_{i j}$. We claim that $\hat{M}_{i j}>0$ on $0 \leq t \leq \delta$ for any $\epsilon>0$. Then letting $\epsilon \rightarrow 0$ will finish the proof.

If $\hat{M}_{i j}$ acquires a null eigenvector $v^{i}$ of unit length at some point $x_{0} \in \partial M$ at time $t_{0}$, i.e.

$$
\hat{M}_{i j} v^{i}=0
$$

for $\forall j$. We also denote the parallel extension of $v^{i}$ to a neighborhood of $x_{\circ}$ as $v^{i}$. Let $f=\hat{M}_{i j} v^{i} v^{j}$, then $f\left(x_{0}, t_{0}\right)=0$ Then we know that $f$ is well-defined in a neighborhood of $x_{\circ}$ and we have

$$
\frac{\partial f}{\partial t}=\epsilon+\Delta f+N_{i j} v^{i} v^{j}
$$

Define $g=f-\eta v$, where $\eta$ is a constant to be chosen later,

$$
v=e^{-\alpha\left[r^{2}+\left(t-t_{0}\right)^{2}\right]}-e^{-\alpha R^{2}}
$$

$R$ is the radius of a small disk $B$ which is centered at $\left(x_{1}, t_{1}\right)$ and is tangent to $\partial M$ at $\left(x_{0}, t_{0}\right)$, and $r=\operatorname{dist}\left(x, x_{1}\right)$. Now let $\hat{B}$ be a small disk centered at $\left(x_{0}, t_{0}\right)$ with radius less than $\frac{1}{2} R$. We also denot $D=B \cap \hat{B}, \Gamma=\partial \hat{B} \cap B$, and $\Gamma^{\prime}=\partial B \cap \hat{B}$.

Then we have on $D$ that

$$
\frac{\partial v}{\partial t}=-2 \alpha\left(t-t_{0}\right) e^{-\alpha\left[r^{2}+\left(t-t_{0}\right)^{2}\right]}
$$

$$
\begin{aligned}
\Delta v=\nabla\left[-2 \alpha r \nabla r e^{-\alpha\left[r^{2}+\left(t-t_{0}\right)^{2}\right]}\right] & \\
& =\left[-2 \alpha r \Delta r+4 \alpha^{2} r^{2}\right] e^{-\alpha\left[r^{2}+\left(t-t_{0}\right)^{2}\right]}
\end{aligned}
$$


By comparison theorem, we have

$$
\Delta r \leq \frac{n-1}{r}(1+k r)
$$

We also notice that $r^{2} \geq \rho^{2}$ on $D$ and $\left|t-t_{0}\right| \leq \rho^{\prime}$ for some constant $\frac{1}{2} R<\rho<R$ and $0<\rho^{\prime}<R$.

Therefore, we see that

$$
\begin{aligned}
\left(\frac{\partial}{\partial t}-\Delta\right) v & \leq 2 \alpha\left[-2 \alpha r^{2}+(n-1)(1+k r)+R\right] e^{-\alpha\left[r^{2}+\left(t-t_{0}\right)^{2}\right]} \\
& \leq 2 \alpha\left[-2 \alpha \rho^{2}+(n-1)(1+k R)+R\right] e^{-\alpha\left[r^{2}+\left(t-t_{0}\right)^{2}\right]}<0
\end{aligned}
$$

for $\alpha$ large enough.

So

$$
\left(\frac{\partial}{\partial t}-\Delta\right) g>\epsilon+N_{i j} v^{i} v^{j}
$$

Since $\hat{M}_{i j} v^{i} v^{j}=0$ by null-eigenvector condition, and

$$
N_{i j} v^{i} v^{j} \geq-C \epsilon \delta
$$

at $\left(x_{0}, t_{0}\right)$ for some constant $\mathrm{C}$ mentioned before, we have

$$
\left(\frac{\partial}{\partial t}-\Delta\right) g>0
$$

at $\left(x_{0}, t_{0}\right)$ if $C \delta<1$.

So $\left(\frac{\partial}{\partial t}-\Delta\right) g>0$ in a small neighborhood $D^{\prime}$ of $\left(x_{0}, t_{0}\right)$.

Also, we know that from the fact that $f\left(x_{0}, t_{0}\right)=0, f>0$ in $D-\left\{x_{0}, t_{0}\right\}$, so $f \geq \eta^{\prime}$ on $\Gamma$. So by choosing $\eta>0$ small enough we have $g \geq 0$ on $\Gamma$. Similarly, by choice of $\Gamma^{\prime}$, we know $g=f>0$ except at $\left(x_{0}, t_{0}\right)$. Hence the maximum principle tells us that

$$
g \geq 0
$$

in $D^{\prime}$ and the minimum occurs only at the point $\left(x_{0}, t_{0}\right)$. The Hopf maximum principle gives

$$
\left.\frac{\partial g}{\partial n}\right|_{\left(x_{0}, t_{0}\right)} \leq 0
$$

with respect to the outward normal. So we obtain

$$
\left.\frac{\partial f}{\partial n}\right|_{\left(x_{0}, t_{0}\right)} \leq\left.\eta \frac{\partial v}{\partial n}\right|_{\left(x_{0}, t_{0}\right)}<0
$$


with respect to the outward normal vector to $\partial M$.

The above inequality immediately implies that

$$
\left.\left(\frac{\partial}{\partial n} M_{i j}\right) v^{i} v^{j}\right|_{\left(x_{0}, t_{0}\right)}<0
$$

which contradicts our assumption. Hence the proof is finished.

Lemma 4.1 immdiately implies that the condition $\alpha g_{i j} \leq R_{i j} \leq \beta g_{i j}$ will be preserved under the Ricci flow.

Actually, if we assume that the evolution equation has a solution on the interval $0 \leq t<T$, we have the following lemma:

Lemma 4.2. If $\varepsilon R g_{i j} \leq R_{i j} \leq \beta R g_{i j}$ for some constants $\varepsilon$ and $\beta$ with $0<\varepsilon \leq \frac{1}{3}<\beta<1$ at $t=0$, then both conditions continue to hold on $0 \leq t<T$.

The proof of this lemma is basically the same as in [Ham]. We apply the Hopf maximum principle with

$$
\begin{gathered}
M_{i j}=\beta g_{i j}-\frac{R_{i j}}{R} \\
u^{k}=\frac{2}{R} \nabla^{k} R \\
N_{i j}=\left(\frac{R Q_{i j}+2 S R_{i j}}{R^{2}}\right)-2 \beta R_{i j}
\end{gathered}
$$

where $S=\mid$ Ric $\left.\right|^{2}$ and tensor $Q_{i j}=6 R_{i}^{k} R_{j}^{k}-3 R R_{i j}+\left(R^{2}-2 S\right) g_{i j}$.

We only need to check that $u^{k} \nabla_{k} M_{i j}$ satisfies the requirement in Lemma 4.1. It is easy to see that because the tangential derivative of $M_{i j}$ equals to zero at its extremun and the normal derivative is zero due to the fact that $R_{n}=0, R_{\alpha \beta ; n}=0$ and $R_{n n ; n}=0$.

In the following we are going to establish a key estimate which is called the 'Pinching estimate'. We need an evolution equation.

Lemma 4.3. Let $f=\frac{S-\frac{1}{3} R^{2}}{R^{\gamma}}$, then we have

$$
\text { (4.65) } \begin{aligned}
\frac{\partial f}{\partial t}=\Delta f & +\frac{2(\gamma-1)}{R} g^{p q} \nabla_{p} R \nabla_{q} f-\frac{2}{R^{\gamma+2}}\left|R \nabla_{i} R_{j k}-\nabla_{i} R R_{j k}\right|^{2} \\
& -\frac{(2-\gamma)(\gamma-1)}{R^{\gamma+2}}\left(S-\frac{1}{3} R^{2}\right)\left|\nabla_{i} R\right|^{2} \\
& +\frac{2}{R^{\gamma+1}}\left[(2-\gamma) S\left(S-\frac{1}{3} R^{2}\right)-2 P\right]
\end{aligned}
$$


where $P=S^{2}+R(C-T), T=R^{i j} R_{i}^{k} R_{j}^{k}$ and $C=\frac{1}{2} Q_{i j} R^{i j}$.

Hamilton proved that

Lemma 4.4. If $R>0$ and $R_{i j} \geq \varepsilon R g_{i j}$, then $P \geq \varepsilon^{2} S\left(S-\frac{1}{3} R^{2}\right)$.

We can choose $\delta>0$ such that $\delta \leq 2 \varepsilon^{2}$, and let $\gamma=2-\delta, f=\frac{S-\frac{1}{3} R^{2}}{R^{\gamma}}$ so that we have the following

$$
\frac{\partial f}{\partial t} \leq \triangle f+u^{k} \nabla_{k} f
$$

where $u^{k}=\frac{2(\gamma-1)}{R} \nabla^{k} R$. Since we have our boundary estimates on the curvature quantities, we know that the maximum of $f$ cannot be achieved for $t>0$, because that will imply that $u^{k} \nabla_{k} f$ vanish at that extrem point on the boundary and hence the Hopf maximum principle says that $\nabla_{n} f<0$ which contradicts our computation that $\nabla_{n} f=0$. Therefore, we have proved the following lemma:

Lemma 4.5. We can find constants $\delta>0$ and $C<\infty$ depending only on the initial metric such that on $0 \leq t<T$ we have

$$
S-\frac{1}{3} R^{2} \leq C R^{2-\delta}
$$

From Lemma 4.5, we can establish an evolution inequality which is also derived in [Ham].

Lemma 4.6. Let $F=|\nabla R|^{2} / R-\eta R^{2}+168\left(S-\frac{1}{3} R^{2}\right)$. Then for any $\eta$ with $0<\eta \leq \frac{1}{3}$ we can find a constant $C(\eta)$ depending only on $\eta$ and the initial value of the metric at $t=0$ such that

$$
\frac{\partial F}{\partial t} \leq \triangle F+C(\eta)
$$

It is easy to see from the evolution equation for scalar curvature $S$ that

Lemma 4.7. If $R \geq R_{\text {min }}$ at time $t=0$ for constant $R_{\min }$, then $T \leq \frac{3}{2 R_{\min }}$.

Now Lemma 4.6 immdiately implies $\max F_{t} \leq \max F_{0}+C(\eta) t$ and hence $F \leq C(\eta)$ for some constant $C(\eta)$ depending only on $\eta>0$ and the initial metric. Therefore, we have

$$
|\nabla R|^{2} \leq \eta R^{3}+C(\eta) R \leq \eta R^{3}+\hat{C}(\eta)
$$


since $\eta$ is arbitrary. We have proved

Theorem 3. For any $\eta>0$, we have constant $C(\eta)$ depending only on $\eta$ and initial metric, such that on $0 \leq t<T$, the following estimate holds

$$
|\nabla R|^{2} \leq \eta R^{3}+C(\eta)
$$

Similarly, we can also follow Hamilton [Ham] to show the following Lemma 4.8. The evolution equation

$$
\frac{\partial g}{\partial t}=-2 \text { Ric }
$$

has a solution on a maximal time interval $0 \leq t<T$. If $T<\infty$, then $\max _{M}\left|R_{i j k l}\right|^{2} \rightarrow \infty$ as $t \rightarrow T$.

In particular, since $R^{2} \geq S$, so $R_{\text {max }} \rightarrow \infty$ as $t \rightarrow T$. By equation (4.67), we know that for every $\eta>0$ we can find a constant $C(\eta)$ with

$$
|\nabla R| \leq \frac{1}{2} \eta^{2} R^{3 / 2}+C(\eta)
$$

on $0 \leq t<T$. Since $\lim _{t \rightarrow T} R_{\text {max }} \rightarrow+\infty$, we can find $T_{0}$ with

$$
C(\eta) \leq \frac{1}{2} \eta^{2} R_{\max }^{3 / 2}
$$

for $T_{0} \leq t<T$. Then $|\nabla R| \leq \eta^{2} R_{\max }^{3 / 2}$ for $t \geq T_{0}$.

Let $x \in M$ such that $R(x)=R_{\max }$, then any geodesic out of $\mathrm{x}$ of length at most $s=\frac{1}{\eta} R_{\max } 1 / 2$ we have $R \geq(1-\eta) R_{\max }$. By Meyers theorem, we know if $\eta$ is small enough then all the geodesics of length greater than $s$ include the entire manifold $M$ and hence

$$
R_{\min } \geq(1-\eta) R_{\max }
$$

Therefore we have proved

Lemma 4.9.

$$
\lim _{t \rightarrow T} \frac{R_{\max }}{R_{\min }}=1
$$

We also quote the following without proof (see [Ham] for details).

Lemma 4.10. We have

$$
\int_{0}^{T} R_{\max } d t=\infty
$$


Lemma 4.11.

$$
\int_{0}^{T} r d t=\infty
$$

where $r=\frac{1}{V_{t}} \int_{M} d \omega_{t}$ and $V_{t}$ is the volume of $g$.

Now the Lemma 4.5 implies that

$$
S / R^{2}-\frac{1}{3} \leq C R^{-\delta} \rightarrow 0 .
$$

In order to prove the convergence, we need to normalize the Ricci flow and we study the following normalized evolution equation:

$$
\frac{\partial g}{\partial t}=-2 \text { Ric }+\frac{2}{3} r g .
$$

It can be easily seen that this normalized equation have solution on $0 \leq t<T$ with $T=\infty$ and

$$
\begin{gathered}
0<\varepsilon \leq R_{\text {min }} \leq R_{\text {max }} \leq C \\
\varepsilon R g_{i j} \leq R_{i j} \leq R g_{i j} \\
\lim _{t \rightarrow \infty} \frac{R_{\text {max }}}{R_{\text {min }}}=0 \\
\lim _{t \rightarrow \infty} \frac{S-\frac{1}{3} R^{2}}{R^{2}}=0 .
\end{gathered}
$$

Actually, we know that Equation (4.67) also holds for $\gamma=2$. We can also check that there is a constant $\delta>0$

$$
\frac{4 P}{R^{3}} \geq 4 \varepsilon^{2} S f / R^{3} \geq 4 \varepsilon^{2} f / 3 R \geq \delta f
$$

and hence $f=\frac{S-\frac{1}{3} R^{2}}{R^{2}}$ satisfies

$$
\frac{\partial f}{\partial t} \leq \Delta f+u^{k} \nabla_{k} f-\delta f
$$

with $u^{k}=\frac{2 \nabla^{k} R}{R}$.

The maximum principle enables us to conclude again that

$$
f \leq C e^{-\delta t}
$$

and hence

Theorem 4. We can find constants $C<\infty$ and $\delta>0$ such that

$$
S-\frac{1}{3} R^{2} \leq C e^{-\delta t} .
$$


Now we

\section{Corollary 1.}

$$
\left|R_{i j}-\frac{1}{3} R g_{i j}\right| \leq C e^{-\delta t}
$$

If we let

$$
F=\frac{|\nabla R|^{2}}{R}+168\left(S-\frac{1}{3} R^{2}\right)
$$

then we have

$$
\frac{\partial F}{\partial t} \leq \triangle F+672 R\left(S-\frac{1}{3} R^{2}\right)-\frac{4}{3} r F .
$$

Estimate (4.68) tells us that

$$
\frac{\partial F}{\partial t} \leq \triangle F+C e^{-\delta t}-\delta F
$$

for some $C<\infty, \delta>0$, since $R \leq C$ and $r \geq R_{\text {min }} \geq \varepsilon>0$. By the Hopf maximum principle we have $F \leq C(1+t) e^{-\delta t}$ and this proves that

Corollary 2. We can find constants $C<\infty$ and $\delta>0$ such that

$$
R_{\max }-R_{\min } \leq C e^{-\delta t}
$$

The last two corollaries immediately give us

\section{Corollary 3.}

$$
\left|R_{i j}-\frac{1}{3} r g_{i j}\right| \leq C e^{-\delta t}
$$

Therefore we have obtained the pinching of the Ricci curvature and scalar curvature. Now the rest of the proof for our long time existence follows exactly the same as in [Ham].

\section{References}

[Ham] R.S. Hamilton, Three-manifolds with positive Ricci curvature, J. Differential Geometry, 17 (1982), 255-306.

[Lady] O. Ladyzenskaja, V. Solonnikov and N. Uralceva, Linear and Quasilinear Equations of Parabolic Type, Translations of Math Monographs, Vol. 23 (1968).

[Sc] R. Schoen, A report on some recent progress on nonlinear probblems in geometry, 1991, preprint.

[Shi] W. Shi, Deforming the metric on complete Riemannian manifolds, J. Diff. Geom., 30 (1989), 223-301. 
[Sim] J. Simons, Minimal varieties in Riemannian manifolds, Ann. of Math., (2) 88 (1968), 62-105.

Received September 15, 1993.

Texas A \& M UNIVERSiTy

College Station, TX 77843 



\title{
PACIFIC JOURNAL OF MATHEMATICS
}

Founded by

\author{
$\begin{array}{ll}\text { E. F. Beckenbach (1906-1982) F. Wolf (1904-1989) } & \text { F }\end{array}$
}

EDITORS

Sun-Yung A. Chang (Managing Editor) Robert Finn

University of California

Los Angeles, CA 90095-1555

pacific@math.ucla.edu

F. Michael Christ

University of California

Los Angeles, CA 90095-1555

christ@math.ucla.edu

Nicholas Ercolani

University of Arizona

Tucson, AZ 85721

ercolani@math.arizona.edu
Stanford University

Stanford, CA 94305

finn@gauss.stanford.edu

Steven Kerckhoff

Stanford University

Stanford, CA 94305

spk@gauss.stanford.edu

Martin Scharlemann

University of California

Santa Barbara, CA 93106

mgscharl@math.ucsb.edu
Gang Tian

Massachusettes Institute of Technology

Cambridge, MA 02139

tian@math.mit.edu

\section{S. Varadarajan}

University of California

Los Angeles, CA 90095-1555

vsv@math.ucla.edu

Dan Voiculescu

University of California

Berkeley, CA 94720

dvv@math.berkeley.edu

\section{SUPPORTING INSTITUTIONS}

\author{
CALIFORNIA INSTITUTE OF TECHNOLOGY \\ NEW MEXICO STATE UNIVERSITY \\ OREGON STATE UNIVERSITY \\ STANFORD UNIVERSITY \\ UNIVERSITY OF ARIZONA \\ UNIVERSITY OF BRITISH COLUMBIA \\ UNIVERSITY OF CALIFORNIA \\ UNIVERSITY OF HAWAII
}

\author{
UNIVERSITY OF MONTANA \\ UNIVERSITY OF NEVADA, RENO \\ UNIVERSITY OF OREGON \\ UNIVERSITY OF SOUTHERN CALIFORNIA \\ UNIVERSITY OF UTAH \\ UNIVERSITY OF WASHINGTON \\ WASHINGTON STATE UNIVERSITY
}

The supporting Institutions listed above contribute to the cost of publication of this Journal, but they are not owners or publishers and have no responsibility for its contents or policies.

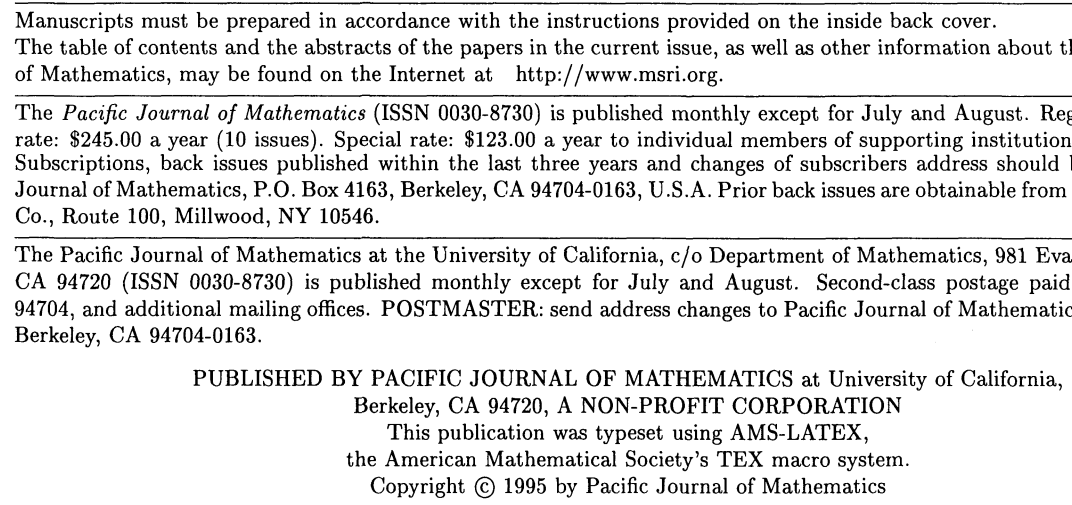




\section{PACIFIC JOURNAL OF MATHEMATICS}

\section{Volume $173 \quad$ No. $1 \quad$ March 1996}

Isometric immersions of $H_{1}^{n}$ into $H_{1}^{n+1}$

KINETSU ABE

Rotationally symmetric hypersurfaces with prescribed mean curvature

MARIE-FRANÇOISE BIDAUT-VÉRON

The covers of a Noetherian module

JIAN-JUN CHUAI

On the odd primary cohomology of higher projective planes

MARK FoSKEY and MiCHAEL DAVID SLACK

Unit indices of some imaginary composite quadratic fields. II

MiKinito HiRABAYASHI

Mixed automorphic vector bundles on Shimura varieties

MIN Ho LeE

Trace ideal criteria for Toeplitz and Hankel operators on the weighted Bergman

spaces with exponential type weights

PENG LIN and RICHARD ROCHBERG

On quadratic reciprocity over function fields

KATHY DONOVAN MERRILL and LYNNE WALLING

$\left(A_{2}\right)$-conditions and Carleson inequalities in Bergman spaces

TAKAHIKO NAKAZI and MASAHIRO YAMADA

A note on a paper of E. Boasso and A. Larotonda: "A spectral theory for solvable Lie 173 algebras of operators"
C. Отт

Tensor products with anisotropic principal series representations of free groups

Carlo Pensavalle and Tim Steger

On Ricci deformation of a Riemannian metric on manifold with boundary

YING SHEN

The Weyl quantization of Poisson $S U(2)$

Albert Jeu-Liang Sheu

Weyl's law for $S L(3, \mathbb{Z}) \backslash S L(3, \mathbb{R}) / S O(3, \mathbb{R})$

ERIC GeORge Stade and Dorothy IRENe Wallace (ANDREOli)

Minimal hyperspheres in two-point homogeneous spaces

PER TOMTER

Subalgebras of little Lipschitz algebras

NiKOLAI ISAAC WEAVER 